$\int \begin{array}{lr}\frac{\text { Journal of Applied Mathematics and Computational Mechanics 2018, 17(3), 53-60 }}{2} & \text { p-ISSN 2299-9965 } \\ \text { e-ISSN 2353-0588 }\end{array}$

\title{
IDENTIFICATION OF BRAINS TISSUE HYPERELASTIC PARAMETERS
}

\author{
Wackaw Kuś, Martyna Poręba-Sebastjan \\ Institute of Mechanics and Computational Engineering \\ Silesian University of Technology, Gliwice, Poland \\ waclaw.kus@polsl.pl,martyna.poreba@student.polsl.pl
}

Received: 9 May 2018; Accepted: 23 October 2018

\begin{abstract}
The goal of the paper is to correlate real brain deflection with its numerical model as the 3D model of a fragment of the brain and suction pipe. The model is analyzed with the Finite Element Method with use of Ansys software. The brain tissue can undergo large strains, which is why it is described by a hyperelastic material. The Mooney-Rivlin material model is used for numerical analyzes. The inverse problem is solved with use of optimization Non-Linear Programming by Quadratic Lagrangian (NLPQL).
\end{abstract}

MSC 2010: 92C10, $74 S 05$

Keywords: inverse problem, biomechanics, brain tissue

\section{Introduction}

The brain is the most complex human organ and its exploration is still of interest to many researchers in various fields of science. Nowadays, despite increasing safety rules, there is still a large number of deaths from brain injury. Therefore, an attempt to reflect the real behavior of the brain tissue using numerical analyzes, will allow the elimination unforeseen tissue damage. This is of great importance above all in dynamic analyzes during injures occurring at the car collisions, accidents and sports contacts. To simulate the real behavior of the brain tissue, it is necessary to choose the appropriate parameters of the hyperelastic material model.

The brain tissue shows the ability to sustain large deformations similar to rubber materials. For this reason, hyperelastic material properties should be assigned but it is important to choose the right material model. Hyperelastic models behave nonlinearly and can be almost or completely incompressible. The most common hyperelastic material models used for brain tissue modelling are Arruda-Boyce, Gent, Mooney-Rivlin, Neo-Hookean, Ogden, Yeoh, and compressible materials include Hyperfoam, Blatz-Ko [1-5]. 


\section{Description of hyperelastic models used for tissue modelling}

Hyperelastic materials can undergo elastic deformations while retaining their original properties. During deformation, material models behave in a non-linear fashion and deformations are not directly proportional to the load. The relationship between deformations and stresses or strains and the energy of hyperelastic bodies is described by means of constitutive equations. The equations for mechanical energy balance are used to determine these equations $[1,2,5]$.

Models of hyperelastic materials are isotropic and constant in relation to temperature. It is believed that these materials are almost or completely incompressible and that the thermal expansion in the material is isotropic $[1,2,5]$.

The very behaviour of hyperelastic materials is supported by software through shell, flat and volume elements [1].

There is a big problem with the appropriate selection of the hyperelastic model. In the case of almost or incompressible materials, the user must choose between phenomenological and micromechanical models, invariants of strain and the main stretching models:

a) the Neo-Hookean is the simplest material model and good at the beginning of the verification of the incompressible model due to the small number of the parameters;

b) the Mooney-Rivlin is one of the most popular hyperelastic models, although it is not suitable for capturing the stiffening effect;

c) the Yeoh model proposes to bypass the second parametric invariant because it is more difficult to measure and provides a less accurate match to the limited test data;

d) the Ogden is based on the main stretching, usually providing a much better curve fit. For this reason, sometimes it increases the complexity of calculations $[1,2,4-6]$.

The hyperelastic material models used in this article, have been compared in the Table 1.

Table 1

Comparison of hyperelastic material models $[1,2,4-10]$

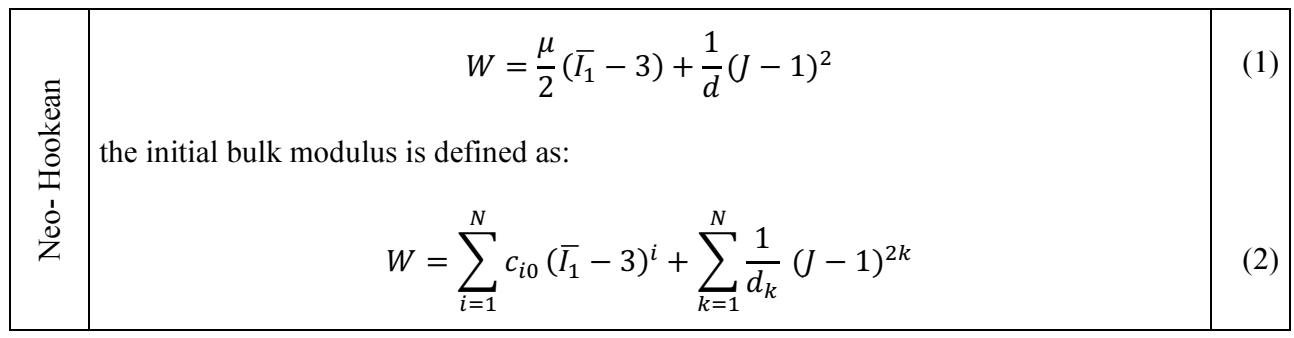


- 2-parameters Mooney-Rivlin option

$$
W=c_{10}\left(\overline{I_{1}}-3\right)+c_{01}\left(\overline{I_{2}}-3\right)+\frac{1}{d}(J-1)^{2}
$$

The initial shear modulus is defined as:

$$
\mu=2\left(c_{10}+c_{01}\right)
$$

And the initial bulk modulus is defined as:

$$
K=\frac{2}{d},\left(\text { where } d=\frac{(1-2 v)}{\left(c_{10}+c_{01}\right)}\right)
$$

- 3-parameters Mooney-Rivlin option

$$
W=c_{10}\left(\overline{I_{1}}-3\right)+c_{01}\left(\overline{I_{2}}-3\right)+c_{11}\left(\overline{I_{1}}-3\right)\left(\overline{I_{2}}-3\right)+\frac{1}{d}(J-1)^{2}
$$

- 5-parameters Mooney-Rivlin option

$$
\begin{aligned}
W=c_{10}\left(\overline{I_{1}}-3\right)+ & c_{01}\left(\overline{I_{2}}-3\right)+c_{20}\left(\overline{I_{1}}-3\right)^{2}+c_{11}\left(\overline{I_{1}}-3\right)\left(\overline{I_{2}}-3\right)+c_{02}\left(\overline{I_{2}}-3\right)^{2} \\
& +\frac{1}{d}(J-1)^{2}
\end{aligned}
$$

- 9-parameters Mooney-Rivlin option

$$
\begin{aligned}
W=c_{10}\left(\overline{I_{1}}-3\right)+ & c_{01}\left(\overline{I_{2}}-3\right)+c_{20}\left(\overline{I_{1}}-3\right)^{2}+c_{11}\left(\overline{I_{1}}-3\right)\left(\overline{I_{2}}-3\right)+c_{02}\left(\overline{I_{2}}-3\right)^{2} \\
& +c_{30}\left(\overline{I_{1}}-3\right)^{3}+c_{21}\left(\overline{I_{1}}-3\right)^{2}\left(\overline{I_{2}}-3\right)+c_{12}\left(\overline{I_{1}}-3\right)\left(\overline{I_{2}}-3\right)^{2} \\
& +c_{03}\left(\overline{I_{2}}-3\right)^{3}+\frac{1}{d}(J-1)^{2}
\end{aligned}
$$

- 1-parameter Yeoh option

$$
W=\sum_{i=1}^{N} c_{i 0}\left(\overline{I_{1}}-3\right)^{i}+\sum_{k=1}^{N} \frac{1}{d_{k}}(J-1)^{2 k}
$$

the initial shear modulus is defined as:

$$
\mu=2 c_{10}
$$

the initial bulk modulus is defined as:

$$
K=\frac{2}{d_{1}}
$$

for $\mathrm{N}=1$ the Yeoh model is equivalent to the Neo-Hookean. 


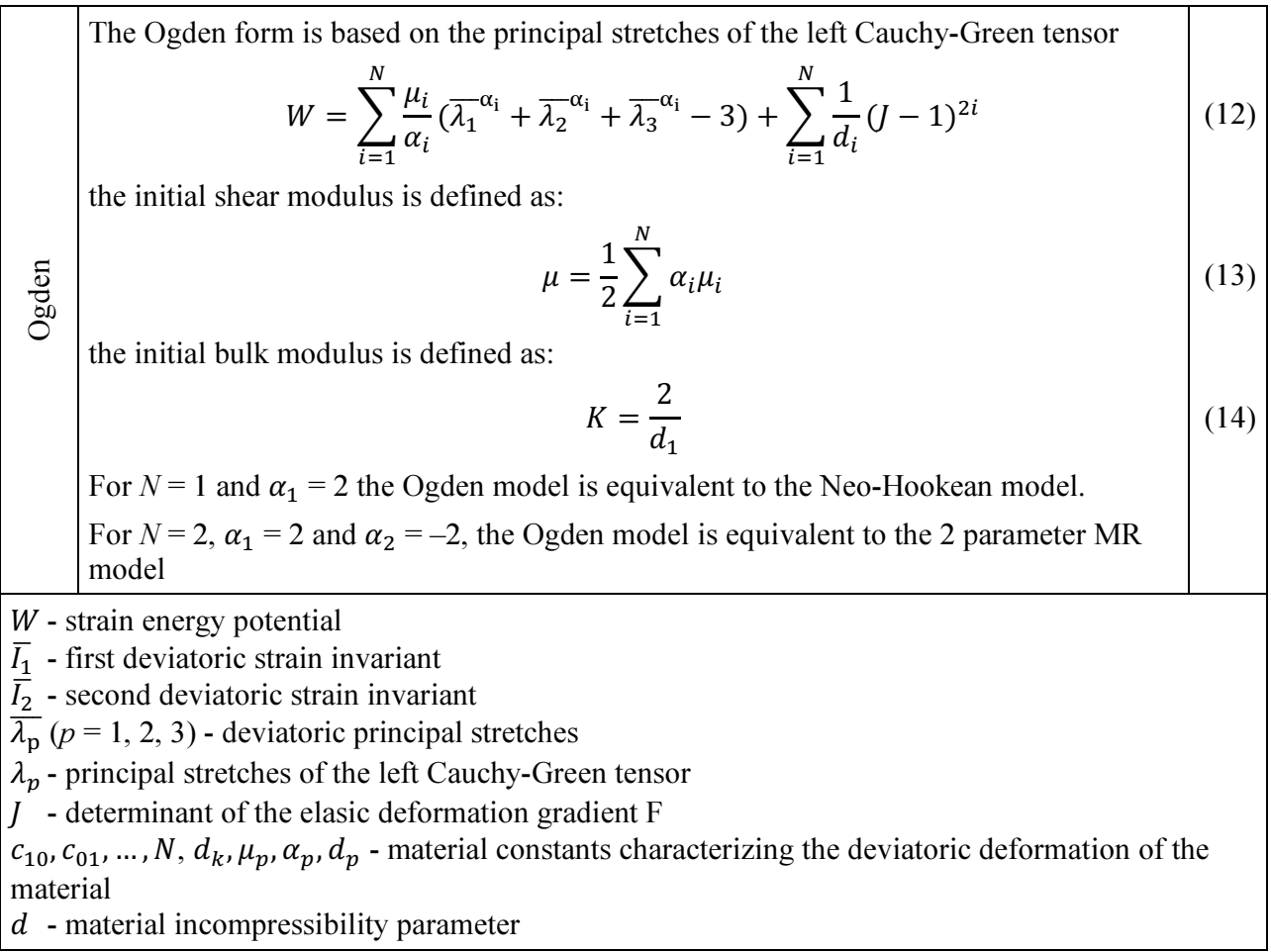

Typical properties of the brain tissue found in the literature are given in Table 2.

Material properties of brain tissue [2-6]

Table 2

\begin{tabular}{|c|c|c|}
\hline Hyperelastic model & Material properties & References \\
\hline Mooney-Rivlin (9-parameters) & $c_{10}=240 \mathrm{~Pa}, c_{30}=3420 \mathrm{~Pa}, v=0.45$ & [2] \\
\hline Neo-Hookean & $c=333.28 \mathrm{~Pa}$ & \\
\hline Mooney-Rivlin & $c_{10}=0.28 \mathrm{~Pa}, c_{20}=333 \mathrm{~Pa}$ & [4] \\
\hline Ogden $\left(3^{\text {rd }}\right.$ order $)$ & $\begin{array}{c}c_{10}=-3543 \mathrm{~Pa}, m_{1}=1, c_{20}=-2723 \mathrm{~Pa}, m_{2}=-1 \\
c_{30}=654 \mathrm{~Pa}, m_{3}=2\end{array}$ & {$[4]$} \\
\hline Yeoh $\left(3^{\text {rd }}\right.$ order $)$ & $c_{10}=185 \mathrm{~Pa}, c_{20}=-601 \mathrm{~Pa}, c_{30}=0.01 \mathrm{~Pa}$ & [3] \\
\hline Mooney-Rivlin (9-parameters) & $c_{10}=62780 \mathrm{~Pa}, c_{20}=8829 \mathrm{~Pa}, c_{30}=6868 \mathrm{~Pa}$ & [6] \\
\hline Ogden $\left(2^{\text {nd }}\right.$ order $)$ & $\begin{aligned} \mu_{1}=-124400 \mathrm{~Pa}, \alpha_{1} & =1.316, \mu_{2}=622500 \mathrm{~Pa}, \\
\alpha_{2} & =1.299\end{aligned}$ & \multirow{5}{*}{ [5] } \\
\hline Ogden $\left(3^{\text {rd }}\right.$ order $)$ & $\begin{array}{c}\mu_{1}=177000 \mathrm{~Pa}, \alpha_{1}=1.055, \mu_{2}=233800 \mathrm{~Pa} \\
\alpha_{2}=1.041, \mu_{3}=325100 \mathrm{~Pa}, \alpha_{3}=0.9889\end{array}$ & \\
\hline Mooney-Rivlin (2-parameters) & $c_{10}=37980 \mathrm{~Pa}, c_{01}=104300 \mathrm{~Pa}$ & \\
\hline Mooney-Rivlin (5-parameters) & $\begin{array}{c}c_{10}=-88410 \mathrm{~Pa}, c_{01}=219700 \mathrm{~Pa}, \\
c_{20}=212200 \mathrm{~Pa}, c_{11}=-290900 \mathrm{~Pa}, c_{02}=78220 \mathrm{~Pa}\end{array}$ & \\
\hline Yeoh $\left(3^{\text {rd }}\right.$ order $)$ & $c_{10}=159500 \mathrm{~Pa}, c_{20}=-6930 \mathrm{~Pa}, c_{30}=403 \mathrm{~Pa}$ & \\
\hline
\end{tabular}




\section{The numerical model of brain tissue and the experiment}

The experiment described in [2] was performed in vivo on human brain. The suction pipe was used to displace brain using small suction pressure. Maximum displacement and corresponding pressure values were recorded. According to [2] the adult patient with brain tumor (outside of the experiment area) was investigated and the procedure was safe without any harm to the patient.

The 3D models of the fragment of the brain and the suction pipe were created in the paper referred above. The numerical simulation allows the measurement of the displacements of the brain tissue for different pressure values. The geometry of the model is shown in Figure 1a. The quarter of the 3D model was analysed, as shown in Figures $1 \mathrm{~b}$ and 1c. The dimensions of the model are taken from [2].

The material properties of hyperelastic axisymmetric model were investigated in [2]. The Mooney-Rivlin 9-parameters model was used with two coefficients (first and third). The goal of the paper is to determine the material properties with use of a more accurate 3D model. The material properties of Mooney-Rivlin model are investigated, but a model more similar to [3-5] models is investigated. The proper values of two parameters $\mathrm{c}_{10}$ and $\mathrm{c}_{20}$ are analysed. The $\mathrm{c}_{30}$ is constant with value 0.01 (low or near zero values like in [3-5]).

The model is analysed with the Finite Element Method with use of Ansys software.

a)

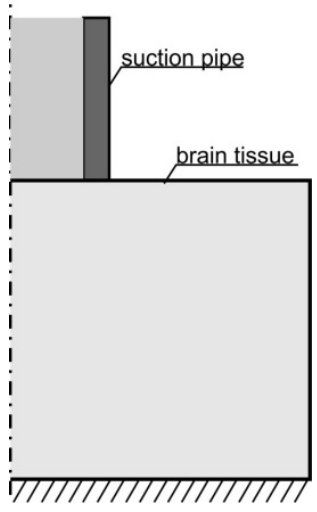

b)

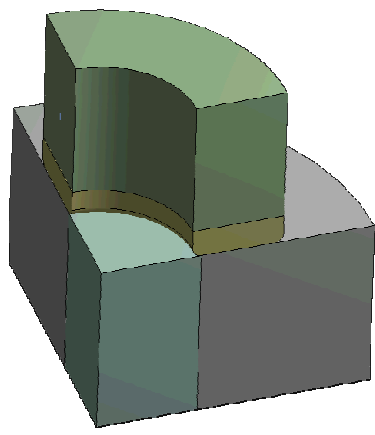

c)

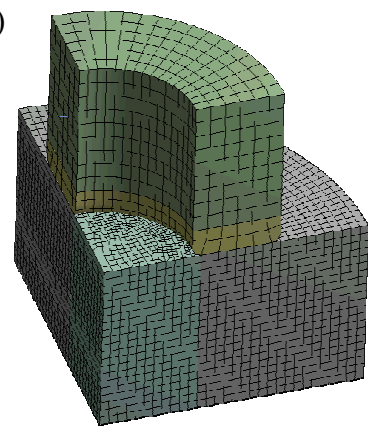

Fig. 1. a) Axisymmetric geometry of the suction pipe and brain tissue,

b) 3D geometry, c) 3D finite element mesh

\section{The inverse problem solution}

The material properties for hyperelastic model were identified using optimization Non-Linear Programming by Quadratic Lagrangian (NLPQL) [11]. The design variables define the material coefficients of hyperelastic model. The objective 
function is defined as a sum of differences between the measured and the computed maximum displacements for a given design vector $\boldsymbol{x}$ :

$$
f(x)=\sqrt{\sum_{i=1}^{n}\left(u_{e i}-u_{n i}\right)^{2}}
$$

where $n$ is the number of recorded displacements during experiment, $u_{e i}$ is the maximum displacement during experiment and $u_{n i}$ is the maximum displacement from numerical model for recorded point $i$. The minimum value of the objective function is known and it is zero. The constraints on design variables are imposed during optimization. The determination of numerical displacements for each design vector leads to direct problem solution, with the use of finite element method (FEM).

\section{Numerical analysis}

The inverse problem was solved taken into account experimental data from [2]. The experiment was performed only once and only five measurements for different suction pressures were recorded. The optimisations were executed from few starting points and leads to material parameters $c_{10}=75$ and $c_{20}=721$. The objective function $f(\boldsymbol{x})$ is equal to $476 \cdot 10^{-6}$. The objective function for material properties taken from [1] is higher and equal to $492 \cdot 10^{-6}$. The evolution of the objective function along with subsequent iterations is shown in Figure 2.

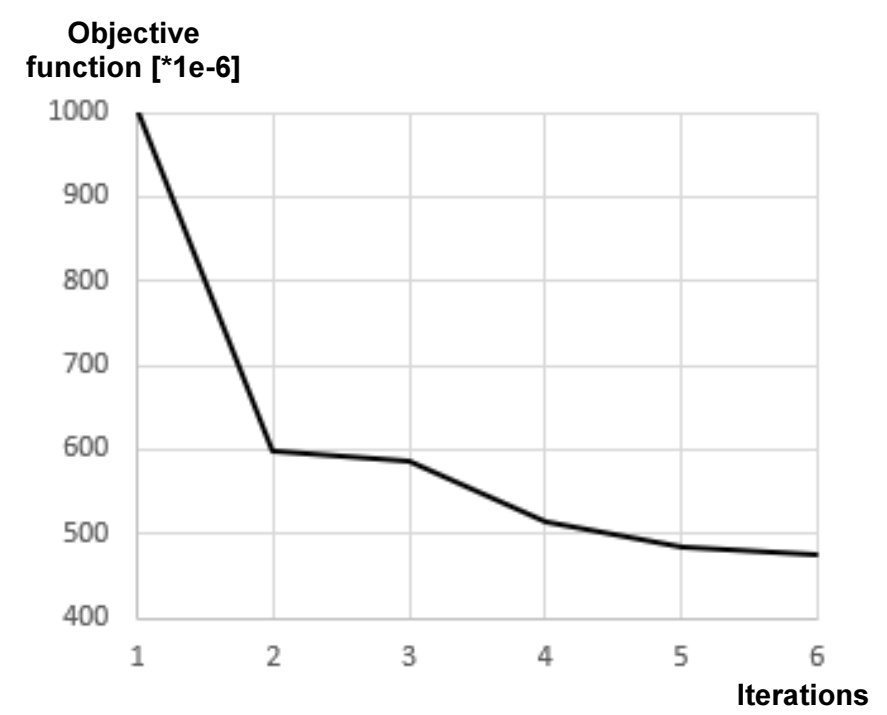

Fig. 2. Evolution of the objective function along with subsequent iterations 
The experimental and numerical maximum displacements for the best solution are shown in Figure 3. The deformation of the brain tissue for the maximum pressure is shown in Figure 4.

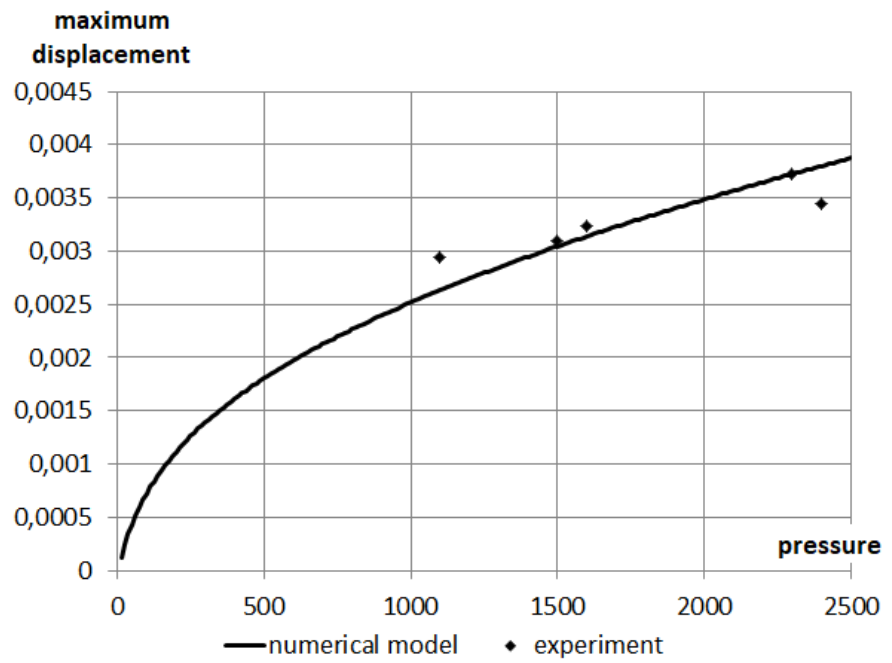

Fig. 3. The maximum displacement from experiment and numerical model in the function of the suction pressure

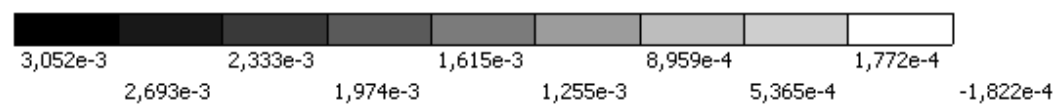

B: Model, Static Structural

Figure

Type: Directional Deformation(Y Axis)

Unit: $m$

Global Coordinate System

Time: 2

Max: $3,052 \mathrm{e}-3$

Min: $-1,822 \mathrm{e}-4$

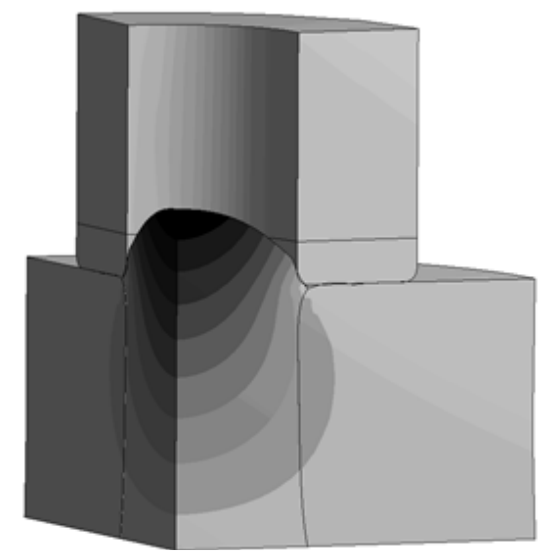

Fig. 4. The deformation of the brain tissue and pipe model

\section{Conclusions}

The material properties depend on the brain region, tissue type and person. The properties were examined for the gray matter. The inverse problem of brain 
tissue material parameters determination was presented in the paper. For this purpose, the 9-parameter Mooney-Rivlin material model was used. The results of the investigations lead to better fit of experimental data than found in literature. The presented method can be applied for other mechanical and biomechanical material properties investigation.

\section{References}

[1] ANSYS $^{\circledR}$ Academic Research Mechanical, Release 18, Help System (2017), ANSYS, Inc.

[2] Schiavone, P., Chassat, F., Boudou, T., Promayon, E., Valdivia, F., \& Payan, Y. (2009). In vivo measurement of human brain elasticity using a light aspiration device. Medical Image Analysis, 13(1), 673-678.

[3] Xing, M. (2015). Tensile behavior and mechanical anisotropy of branched cerebral vasculature within gray matter. Graduate Program in Mechanical and Aerospace Engineering, 1(1), 33-36.

[4] Mihai, L.A., Chin, L., Janmey, P.A., \& Goriely, A. (2015). A comparision of hyperelastic constitutive models applicable to brain and fat tissues, J. R. Soc. Interface, 12, 20150486, 1(1), 3-18.

[5] Rackl, M. (2015). Curve fitting for Ogden, Yeoh and polynomial models. ScilabTEC Conference 2015, 1(1), 1-11.

[6] Budday, S., Nay, R., Rooij, R., Steinmann, P., Wyrobek, T., Ovaert, T.C., \& Kuhl, E. (2015). Mechanical properties of gray and white matter brain tissue by indentation. Journal of Mechanical Behavior of Biomedical Materials, 46(1), 319-329.

[7] Rashid, B., Destrade, M., \& Gilchrist, M.D. (2012). Mechanical characterization of brain tissue in simple shear at dynamic strain rates. Journal of the Mechanical Behavior of Biomedical Materials, 28(1), 73-83.

[8] Prevost, T.P., Balakrishnan, A., Suresh, S., \& Socrate, S. (2010). Biomechanics of brain tissue. Acta Biomaterialia, 7(1), 84-92.

[9] Van Dommelen, J.A.W., van der Sande, T.P.J, Hrapko, M., \& Peters, G.W.M. (2009). Mechanical properties of brain tissue by indentation: interregional variation. Journal of the Mechanical Behavior of Biomedical Materials, 3(1), 160-164.

[10] Kleiven, S. (2002). Finite element modeling of the human head. Department of Aeronautics Royal Institute of Technology, 1(1), 1-37.

[11] Schittkowski, K. (2011). A robust implementation of a sequential quadratic programming algorithm with successive error restoration. Optimization Letters, 5(2), 283-296. 